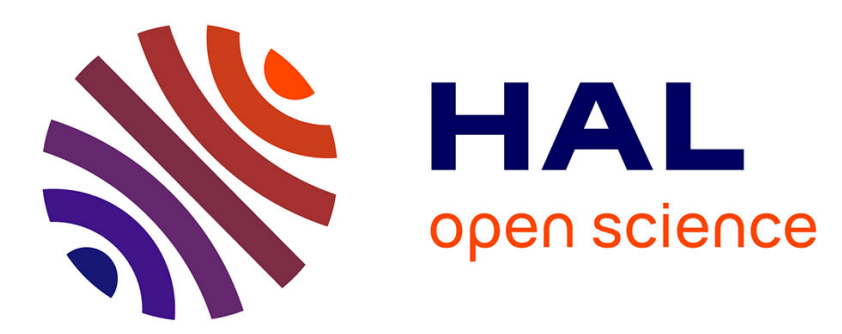

\title{
On the possibility of performing self-calibrated selective $\pi / 2$ pulses in nuclear-magnetic resonance
}

Daniel Abergel, Alain Louis-Joseph, Jean-Yves Lallemand

\section{To cite this version:}

Daniel Abergel, Alain Louis-Joseph, Jean-Yves Lallemand. On the possibility of performing selfcalibrated selective $\pi / 2$ pulses in nuclear-magnetic resonance. Journal of Chemical Physics, 2000, 112 (14), pp.6365-6370. 10.1063/1.481197 . hal-00954907

\section{HAL Id: hal-00954907 \\ https://hal-polytechnique.archives-ouvertes.fr/hal-00954907}

Submitted on 7 Apr 2014

HAL is a multi-disciplinary open access archive for the deposit and dissemination of scientific research documents, whether they are published or not. The documents may come from teaching and research institutions in France or abroad, or from public or private research centers.
L'archive ouverte pluridisciplinaire HAL, est destinée au dépôt et à la diffusion de documents scientifiques de niveau recherche, publiés ou non, émanant des établissements d'enseignement et de recherche français ou étrangers, des laboratoires publics ou privés. 


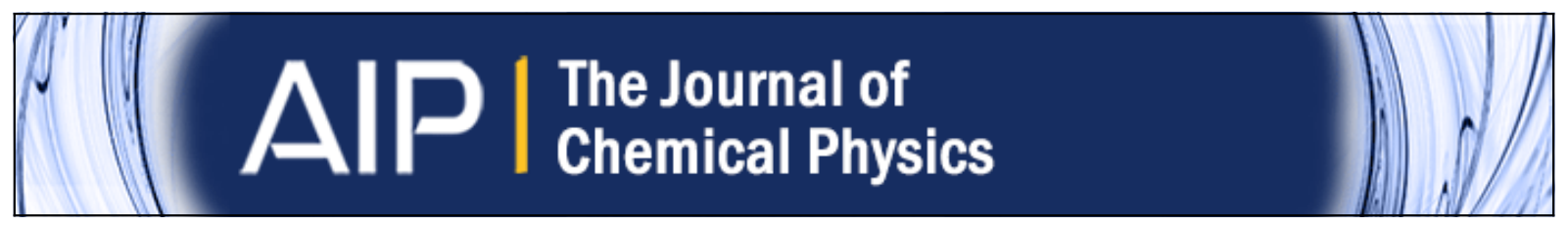

\section{On the possibility of performing self-calibrated selective $/ 2$ pulses in nuclear-magnetic} resonance

Daniel Abergel, Alain Louis-Joseph, and Jean-Yves Lallemand

Citation: The Journal of Chemical Physics 112, 6365 (2000); doi: 10.1063/1.481197

View online: http://dx.doi.org/10.1063/1.481197

View Table of Contents: http://scitation.aip.org/content/aip/journal/jcp/112/14?ver=pdfcov

Published by the AIP Publishing

\section{Articles you may be interested in}

$\mathrm{R} 87 \mathrm{~b}$ and $\mathrm{C} 133 \mathrm{~s}$ nuclear magnetic resonance study of the structural properties of mixed $\mathrm{Rb} \times \mathrm{Cs} 2 \times \mathrm{ZnCl} 4$ ( $\mathrm{x}=0,1$, and 2) crystals

J. Appl. Phys. 107, 093508 (2010); 10.1063/1.3406146

Measurement of $\mathrm{N} 15-\mathrm{T} 1$ relaxation rates in a perdeuterated protein by magic angle spinning solid-state nuclear magnetic resonance spectroscopy

J. Chem. Phys. 128, 052316 (2008); 10.1063/1.2819311

Longitudinal relaxation time detection using a high- T c superconductive quantum interference device magnetometer

J. Appl. Phys. 102, 033914 (2007); 10.1063/1.2767193

First NMR study on a C 60 ternary "superconductor" compound: K 6 C 60 (C 6 H 6 ) 1.5

AIP Conf. Proc. 544, 42 (2000); 10.1063/1.1342464

Quasiparticle properties of $d$-wave superconductors in the vortex state

AIP Conf. Proc. 483, 341 (1999); 10.1063/1.59600

\section{AlP Re-register for Table of Content Alerts}




\title{
On the possibility of performing self-calibrated selective $\pi / 2$ pulses in nuclear-magnetic resonance
}

\author{
Daniel Abergel, Alain Louis-Joseph, and Jean-Yves Lallemand \\ Groupe de RMN, Laboratoire DCSO, Ecole Polytechnique, 91128 Palaiseau Cedex, France
}

(Received 9 November 1999; accepted 7 January 2000)

\begin{abstract}
In this paper, a generalization of the concept of selective "slaved" pulses in NMR spectroscopy introduced recently by the authors is presented. The shape of these pulses is slaved to that of a signal produced by the sample by the action of an electronic feedback loop. The theoretical analysis is based on results of the theory of nonlinear dynamical systems, which predicts the possibility of performing $90^{\circ}$ self-calibrated radiofrequency pulses, through the simultaneous action of a constant radio-frequency field and of a magnetization dependent field of the kind above. Experimental demonstrations show that rotation of the magnetization onto the $x y$-plane is achieved, regardless of its initial position. Moreover, it is shown to remain stable for more than $50 \mathrm{~ms}$, without significant loss of intensity. (C) 2000 American Institute of Physics. [S0021-9606(00)01213-7]
\end{abstract}

\section{INTRODUCTION}

Nonlinear effects in NMR of liquids have become increasingly present in the practice of high-field spectroscopy during the recent years, and since the observation of "multiple quantum"-like effects in 2D spectra of water ${ }^{1}$ the two different phenomena involved, namely, radiation damping ${ }^{2}$ and the demagnetizing field, ${ }^{3}$ have been insistently investigated. Consistent models have lead to satisfactory explanation of anomalous effects observed in high resolution multidimensional experiments in liquids. ${ }^{4-6}$ And due to their increasing magnitude at higher fields, various experimental schemes have been proposed to eliminate them. ${ }^{7,8}$ Demonstration was made that radiation damping could be controlled at will by the use of a simple electronic feedback device. ${ }^{7}$ This provides a means of selectively manipulating the water magnetization. On these grounds a new and original approach was introduced to perform self-calibrated selective $180^{\circ}$ pulses, ${ }^{9}$ which was proven to be efficient for water magnetization inversion and subsequently applied to the study of chemical exchange.

In this paper, we propose a generalization of this approach, which is based on a qualitative analysis of the nonlinear system describing the dynamics of the magnetization in a NMR spectrometer, subject to a radio-frequency field generated by a feedback system of the kind mentioned above, with arbitrary phase and gain. The theory predicts the possibility of generating selective $90^{\circ}$ pulses by the same electronic feedback device. First, the concept of these socalled "slaved pulses" will be recalled. Second, we will present a theoretical description of the problem, which will provide us with a consistent picture of the phenomenon. Finally, experiments performed on a $600 \mathrm{MHz}$ spectrometer will be shown, which demonstrate the validity of the method.

\section{PRINCIPLES OF THE SLAVED PULSE APPROACH}

The basic principle of self-calibrated $180^{\circ}$ selective pulses may be explained as follows. Conventional pulses in
NMR spectroscopy are usually defined in terms of a certain flip angle about a certain axis, which sets the phase of the pulse. However, what one is really aiming at is rather to bring the magnetization from an initial definite position towards a given direction in space. This is achieved by determining the time during which a radio-frequency field of a given magnitude and/or of a given frequency profile should be applied. Problems may arise in practice in complex pulse sequences, for instance, when the state of the magnetization just before a pulse may not coincide with the expected one (this situation is most common when radiation damping is present). In this case an error of this initial state leads to a corresponding error on the state of the magnetization after the pulse. In this respect, even careful calibration of conventional pulses may not guarantee exact knowledge of the final state of the magnetization. This should be distinguished from what is known as pulse imperfection, which refers to the fact that, given an initial state of the magnetization, a certain degree of error in the actual flip angle remains, mostly due to rf field inhomogeneity. The basic idea of self-calibrated pulses $^{9}$ is to use the signal generated by the precessing transverse magnetization of the spins in the detecting coil to generate a feedback signal with a constant phase shift with respect to the former and the intensity of which is proportional to the detected signal. This in turn creates a radio-frequency field with a constant phase with respect to the transverse magnetization and whose magnitude is proportional to it. By controlling the phase of the feedback field, it is possible to create a magnetization-dependent radio-frequency field, which will drive the magnetization towards the $-z$ direction if it is ahead of the magnetization, and back to the $+z$ direction if it lags the transverse magnetization. The latter situation occurs in practice when radiation damping is present, with a phase lag of $-90^{\circ}$. Using an electronic feedback system which was originally designed to allow complete control of radiation damping (i.e., elimination, as well as amplification and inversion) we have shown that it is possible to actually achieve high quality selective inversion of the water 


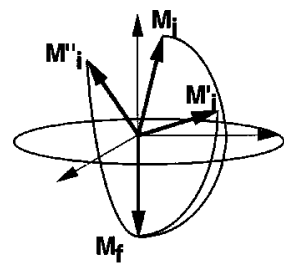

(a)

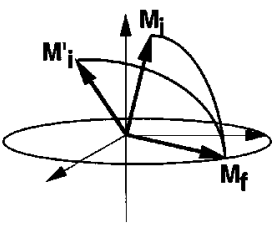

(b)
FIG. 1. In this figure the aim of self-calibrated pulses is schematized. In (a), the case of $180^{\circ}$ pulses (more precisely pulses that drive the magnetization to the $-z$ axis) is shown. In (b), the magnetization is rotated towards the $x y$-plane and remains locked along a desired axis. In both cases, the final state should be independent of the initial position of the magnetization.

magnetization, as already mentioned. The important point is that these $180^{\circ}$ pulses are autocalibrated with respect to phase and amplitude, in the following sense. The feedback field is proportional in magnitude to the transverse magnetization, which becomes zero when the magnetization points towards $-z$ (note that for an inversion pulse on resonance the feedback field is $90^{\circ}$ of phase ahead of the transverse magnetization). It turns out that the magnetization ceases to rotate as soon as it reaches $-z$, so that the magnetization is "locked" on the $z$ axis and points towards $-z$. This is true whatever the initial deviation of the magnetization from the $+z$ direction, and also regardless of the initial phase of the magnetization in the rotating frame, since the feedback field will always remain orthogonal to the transverse magnetization. These points are illustrated in Fig. 1.

The question we wish to address in this paper is wether it is possible to extend this approach to generate $90^{\circ}$ selective pulses. In other words, is it possible to generate controlled pulses that will bring the magnetization along a given axis in the $x y$ plane and maintain it there at long times regardless of the initial position? The fully general answer to this question is not simple, but a theoretical analysis of the problem in terms of fixed points and stability analysis of the differential system governing the dynamics of the magnetization may come up with interesting solutions. Let us remark that in the case of $180^{\circ}$ slaved pulses the magnetization remains in the $-z$ direction once it gets there can be translated into the language of dynamical system theory as the existence of an asymptotic stable fixed point at $\left(0,0,-M_{0}\right)$. This means that the solution of the evolution equations for long times $(t \rightarrow+\infty)$ is stationary and that the magnetization then points towards the south pole. It thus appears as a particular case of the problem at hand, and one may search for such stable fixed points located in the plane, i.e., with $z=0$. This situation cannot occur in the case considered above, where no radiofrequency field other than the feedback field is present, as will be shown in the following, but it will be argued that with the simple use of a weak constant radiofrequency field, and under certain conditions, it can actually be so.

\section{EVOLUTION OF THE MAGNETIZATION UNDER THE ACTION OF A FEEDBACK OF ARBITRARY PHASE: ELEMENTS OF A THEORETICAL TREATMENT}

The evolution of a nonlinear dynamical system is often difficult to picture, and may frequently lead to nonintuitive behavior. Moreover the latter can be difficult to analyze, and a general theory only provides qualitative statements, ${ }^{10}$ so one has to resort to the particular case at hand to sometimes get only a partial understanding of the dynamics. This is the case for the behavior of the magnetization driven by a feedback radio-frequency field. In the following, we propose an elementary theoretical analysis which will lead to quite interesting predictions.

The evolution of the magnetization undergoing the effect of a radio-frequency field and of a radiation dampinggenerated feedback field is given in the rotating frame of coordinates by the following system. In order to keep a lesser number of independent parameters in the dynamic equations, it is convenient to combine the effects of the radiation damping field and the feedback field in a single term,

$$
\begin{aligned}
\dot{M}_{x}= & \delta M_{y}+G M_{z}\left[M_{x} \sin \psi-M_{y} \cos \psi\right]-M_{x} / T_{2}, \\
\dot{M}_{y}= & -\delta M_{x}-\omega_{1} M_{z}+G M_{z}\left[M_{x} \cos \psi+M_{y} \sin \psi\right] \\
& -M_{y} / T_{2}, \\
\dot{M}_{z}= & \omega_{1} M_{y}-G \sin \psi\left[M_{x}^{2}+M_{y}^{2}\right]-\left(M_{z}-M_{0}\right) / T_{1},
\end{aligned}
$$

where $T_{1,2}$ are the longitudinal and transverse relaxation times, respectively, $\delta$ is the frequency shift offset in $\operatorname{rads}^{-1}$, $G$ and $\psi$ are the magnitude and the phase of the combined \{radiation damping + feedback field $\}$ with respect to the transverse magnetization. This combination of fields will be designated simply as the feedback field in the following. The constant radio-frequency intensity, which with no loss of generality is assumed to be parallel to the $x$-axis, is $\omega_{1}$ $=\gamma B_{1}$. The reduced variables are introduced as follows: $t$ $\rightarrow \omega_{1} t, G \rightarrow \lambda=G / \omega_{1}, \delta \rightarrow \delta / \omega_{1}, T_{1,2} \rightarrow \tau_{1,2}=\omega_{1} T_{1,2}$. One thus obtains ${ }^{1}$

$\dot{M}_{x}=\delta M_{y}+\lambda M_{z}\left[M_{x} \sin \psi-M_{y} \cos \psi\right]-M_{x} / \tau_{2}$,

$\dot{M}_{y}=-\delta M_{x}-M_{z}+\lambda M_{z}\left[M_{x} \cos \psi+M_{y} \sin \psi\right]-M_{y} / \tau_{2}$,

$\dot{M}_{z}=M_{y}-\lambda \sin \psi\left[M_{x}^{2}+M_{y}^{2}\right]-\left(M_{z}-M_{0}\right) / \tau_{1}$.

These equations describe the evolution of the magnetization when radiation damping alone is present. Then we have $\psi$ $=-\pi / 2$ and $G=2 \pi \eta Q$, where $Q$ is the quality factor of the probe and $\eta$ is the filling factor. We introduce here a further assumption in neglecting relaxation, so that the magnitude of the magnetization is a constant of the motion, which is easily verified from the relation,

$$
\frac{d}{d t} \mathbf{M}^{2}=2 \mathbf{M} \cdot \frac{d \mathbf{M}}{d t}=0 .
$$

Simplification is obtained by going to the polar coordinates $\left(M_{x}=\sin \theta \cos \varphi, M_{y}=\sin \theta \sin \varphi, M_{z}=\cos \theta\right)$. Equation (3) tells us that the equilibrium magnetization remains equal to its equilibrium value $M_{0}$, which for convenience will be 
taken equal to unity in the following. The equations thus reduce to a set of two coupled equations for the polar angles $\theta$ and $\varphi$ of the magnetization,

$$
\begin{aligned}
& \dot{\theta}=\lambda \sin \psi \sin \theta-\sin \varphi=V_{\theta}(\theta, \varphi), \\
& \dot{\varphi}=\lambda \cos \psi-\delta-\cot \theta \cos \varphi=V_{\varphi}(\theta, \varphi) .
\end{aligned}
$$

In the absence of a radio-frequency field, these equations write

$$
\dot{\theta}=\lambda \sin \psi \sin \theta, \quad \dot{\varphi}=\lambda \cos \psi-\delta .
$$

Here, the equations are decoupled and are easily integrated to give solutions for $\theta$ and $\varphi$. In particular, one obtains

$$
\tan (\theta / 2)=\tan \left(\theta_{0} / 2\right) \exp \left[\lambda \sin \psi\left(t-t_{0}\right)\right],
$$

so that depending on the sign of $\psi, \theta=0, \pi$ is the only fixed point, and that $\theta \rightarrow 0, \pi$ in the limit $t \rightarrow+\infty$, so that $\theta=0, \pi$ is asymptotically stable. Note that for $\psi=0, \theta=\theta_{0}=$ cnst.

With the idea of determining whether it is possible to perform self calibrated pulses of arbitrary pulse length one is interested first in finding the fixed points of the complete dynamical system and secondly in making a stability analysis of these. This is a difficult task in general, but we shall see in that at least some qualitative information can be obtained and physical insight gained from such a study. The fixed points are given by $\dot{\theta}=\dot{\varphi}=0$,

$$
\begin{aligned}
& \lambda \sin \psi \sin \theta-\sin \varphi=0, \\
& \lambda \cos \psi-\delta-\cot \theta \cos \varphi=0 .
\end{aligned}
$$

Nonlinear differential equation theory tells us that the stability of the fixed points of a nonlinear system can be inferred from the stability of the linearized system in a neighborhood of the fixed points. ${ }^{11}$ So one has to study the linearized system,

$$
\begin{aligned}
\frac{d}{d t}\left[\begin{array}{l}
\theta^{\prime} \\
\varphi^{\prime}
\end{array}\right] & =\left[\begin{array}{ll}
\partial V_{\theta}(\theta, \varphi) / \partial \theta & \partial V_{\theta}(\theta, \varphi) / \partial \varphi \\
\partial V_{\varphi}(\theta, \varphi) / \partial \theta & \partial V_{\varphi}(\theta, \varphi) / \partial \varphi
\end{array}\right]_{\theta_{0}, \varphi_{0}}\left[\begin{array}{l}
\theta^{\prime} \\
\varphi^{\prime}
\end{array}\right] \\
& =\partial V\left[\begin{array}{c}
\theta^{\prime} \\
\varphi^{\prime}
\end{array}\right]
\end{aligned}
$$

where $\theta^{\prime}$ and $\varphi^{\prime}$ are the local coordinates near the fixed point $\left(\theta_{0}, \phi_{0}\right)$. In our case, we have

$$
\frac{d}{d t}\left[\begin{array}{c}
\theta^{\prime} \\
\varphi^{\prime}
\end{array}\right]=\left[\begin{array}{cc}
\lambda \sin \psi \cos \theta_{0} & -\cos \varphi_{0} \\
\cos \varphi_{0}\left(1+\cot ^{2} \theta_{0}\right) & \sin \varphi_{0} \cot \theta_{0}
\end{array}\right]\left[\begin{array}{c}
\theta^{\prime} \\
\varphi^{\prime}
\end{array}\right] .
$$

The eigenvalues (or their real part if they are complex) of the linearized system are found to be

$$
\nu_{ \pm}=\frac{1}{2}\left(\sin \varphi_{0} \cot \theta_{0}+\lambda \sin \psi \cos \theta_{0} \pm \sqrt{\Delta}\right),
$$

with

$$
\begin{aligned}
\Delta= & \left(\sin \varphi_{0} \cot \theta_{0}+\lambda \sin \psi \cos \theta_{0}\right)^{2} \\
& -4\left(\lambda \sin \psi \sin \varphi_{0} \cos \theta_{0} \cot \theta_{0}+\frac{\cos ^{2} \varphi_{0}}{\sin ^{2} \theta_{0}}\right) .
\end{aligned}
$$

Stability then requires that the condition $\nu_{ \pm}<0$ on the linearized eigenvalues be fulfilled. Moreover, asymptotic stability means that the fixed point is stable for $t \rightarrow+\infty$. The simple but crucial question addressed in this paper is thus the possibility of performing a simple experiment where an asymptotic fixed point exists in the $x y$-plane. To answer this question, let us focus on a particular case, where the fixed point is near the $x y$-plane, that is, suppose that $\theta_{0}=\pi / 2$ $+\varepsilon$, with $\varepsilon$ a small number. Moreover, let us seek a fixed point with $\varphi_{0}=\pi / 2$. The eigenvalues take the simpler form,

$$
v_{ \pm}=-\tan \varepsilon,-\lambda \sin \psi \sin \varepsilon
$$

and the stability condition thus requires that both $v_{+}$and $v_{-}$ be strictly negative. Therefore, we see that $\theta \in] \pi / 2, \pi[$ and $\psi \in] 0, \pi[$. When expressed in the eigenvector basis of the linearized system, it is clear that the solution tends to $(0,0)$ in the limit $t \rightarrow+\infty$. And in the particular situation envisaged here, the fixed point $\left(\theta_{0}=\pi / 2+\varepsilon, \varphi_{0}=\pi / 2\right)$ is thus defined by

$$
\begin{aligned}
& \sin \theta_{0}=\sin (\pi / 2+\varepsilon)=(\lambda \sin \psi)^{-1}, \\
& \cos \psi=\delta / \lambda .
\end{aligned}
$$

These equations give two necessary conditions of existence of the fixed point, which are $|\lambda \sin \psi| \geqslant 1$ and $|\delta / \lambda| \leqslant 1$.

Now, since $\varepsilon$ is small, the fixed point is near the $x y$ plane. One thus has $\cos \varepsilon \approx 1-\frac{1}{2} \varepsilon^{2} \approx 1 / \lambda \sin \psi$, so that $\varepsilon$ $\approx \sqrt{2(1-1 / \lambda \sin \psi)}$. But since $\sin \psi=-\sqrt{1-\delta^{2} / \lambda^{2}}$, this results in

$$
\varepsilon=\sqrt{2\left(1-1 / \sqrt{\lambda^{2}-\delta^{2}}\right)} .
$$

The latter equation gives the condition for $\lambda$. For instance, for a pulse on resonance $(\delta=0) \lambda=G / \omega_{1}$ is close to unity, with $G>\omega_{1}$. Also, it is noteworthy that one has $\sin \psi$ $\approx 1 / \lambda$. If $\lambda<1$, these equations do not admit a constant stationary solution, and the motion is a precession about a magnetization-dependent radio-frequency magnetic field.

The foregoing discussion has explicitly assumed a fixed point at $\theta_{0}=\pi / 2+\varepsilon$, i.e., close to the $x y$-plane, for small enough values of $\varepsilon$. However, it is important to remark that a fixed point with $\theta=\pi / 2$ cannot be studied in a straightforward manner by the convenient analysis of the linearised system outlined above, since in this case the fixed point is not simple, i.e., the linearized eigenvalues are zero or have zero real part. Indeed, one has $v= \pm i \cos \varphi$, as seen from Eq. (9); then either $\operatorname{Re}(v)=0$ for $\varphi_{0} \neq 0$ or $v_{ \pm}=0$ for $\varphi_{0}=0$ and the linearization theorem does not apply. However, for our purpose we considered $\varepsilon$ a small enough number and yet nonzero, so that one finds a fixed point close enough to the $x$ - $y$-plane, and the above treatment is still reasonable.

At this point of the discussion, the question of the sensitivity to initial conditions should be investigated, since it is crucial to determine whether or not an actual experiment will be likely to take the magnetization to the $x y$-plane, regardless of the initial position of the magnetization. We haven't searched for a systematic answer to this question at this elementary level of analysis, but results from numerical simulations suggest that it is likely to be the case. For sake of illustration, trajectories corresponding to three different initial locations of the magnetization, are shown in Fig. 2. All the trajectories end up at the same fixed point as predicted by the above theory. Remarking that this was also the case for all other initial conditions tested. It should be noted that the 

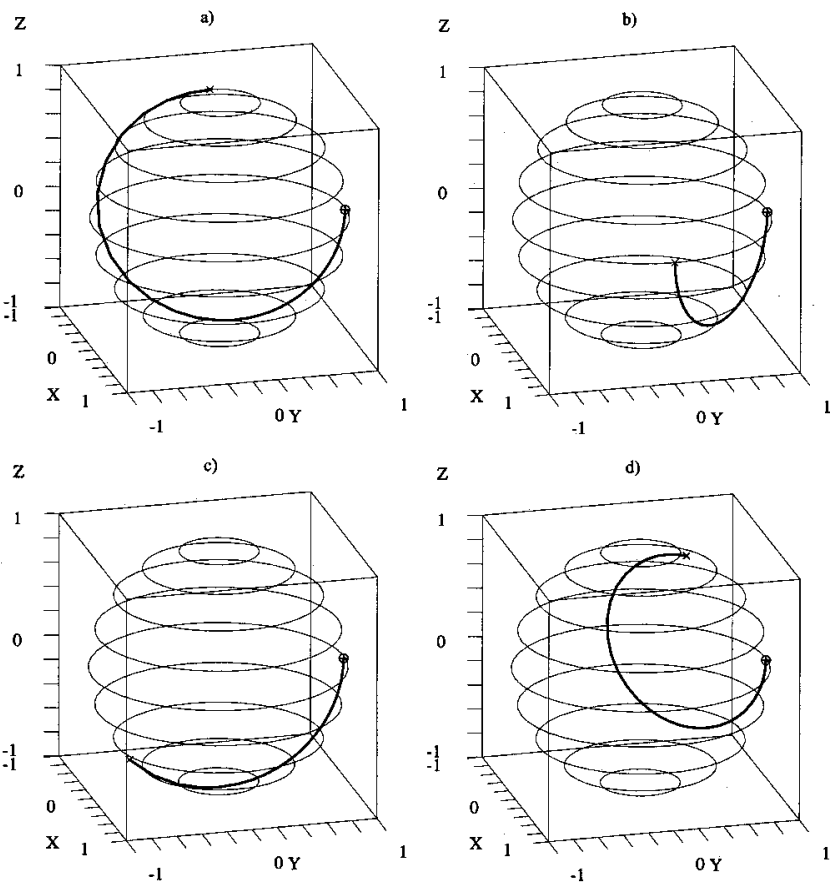

FIG. 2. Probing the basin of attraction of the fixed point of the unrelaxed model. Various initial conditions are shown. (a) $\theta=0.3 \times \pi, \phi=\pi$, (b) $\theta$ $=\pi / 2, \phi=0$, and (c) $\theta=0.75 \times \pi, \phi=-\pi / 2$, (d) $\theta=\pi / 4, \phi=0.75 \times \pi$. The stationary fixed point is reached for each of the initial conditions chosen. Parameters used are $\omega_{1}=300 \times 2 \pi \mathrm{rads}^{-1}, G=300 \times 2 \pi \mathrm{rad} \mathrm{s}^{-1}, \quad \delta=0$. The trajectory is calculated for $50 \mathrm{~ms}$.

parameters used in the numerical calculations of this unrelaxed model were such that $\lambda=G / \omega_{1}=1, \psi=\pi / 2$ and $\delta$ $=0$, which, according to the discussion above, gives credit to the assumption of stability conservation of Eq. (3) during the limiting process $\varepsilon \rightarrow 0$.

These results are promising, but of course the question still remains as to the validity of the above discussion in a more realistic model, where relaxation is taken into account. It is easy to show and important to mention that, strictly speaking, there is no stationary solution for the magnetization having both the required conditions in this case: conserved magnitude (equal to $M_{0}$ ) and pointing in a definite direction in the $x y$-plane. However, simulation of the complete system of equations shows that for certain values of the radio-frequency intensity $\omega_{1}$, resonance offset $\delta$ and feedback amplification and phase $(\lambda, \psi)$, it is possible to drive the magnetization towards the $x y$-plane, where it spirals inwards about an axis with slowly varying orientation. Appropriate conditions were found by investigating regions of the parameter space $\left\{\lambda, \delta, \omega_{1}\right\}$ neighboring the matching conditions found for the unrelaxed case. Results are shown in Fig. 3. It is seen that the magnetization is predicted by approaching the stabilization axis in the $x y$-plane, residing in its neighborhood for a certain period of time, before relaxing at longer times towards an equilibrium value. With the values chosen for the parameters in the simulations, the time needed to reach a quasisteady state is about $20 \mathrm{~ms}$ and it remains aligned along a stable axis in the $x y$-plane for a period of time on the order of $30 \mathrm{~ms}$ [Figs. 3(a)-3(c)]. It is noteworthy that the phase of the stabilization axis is not $\pi / 2$, as in the
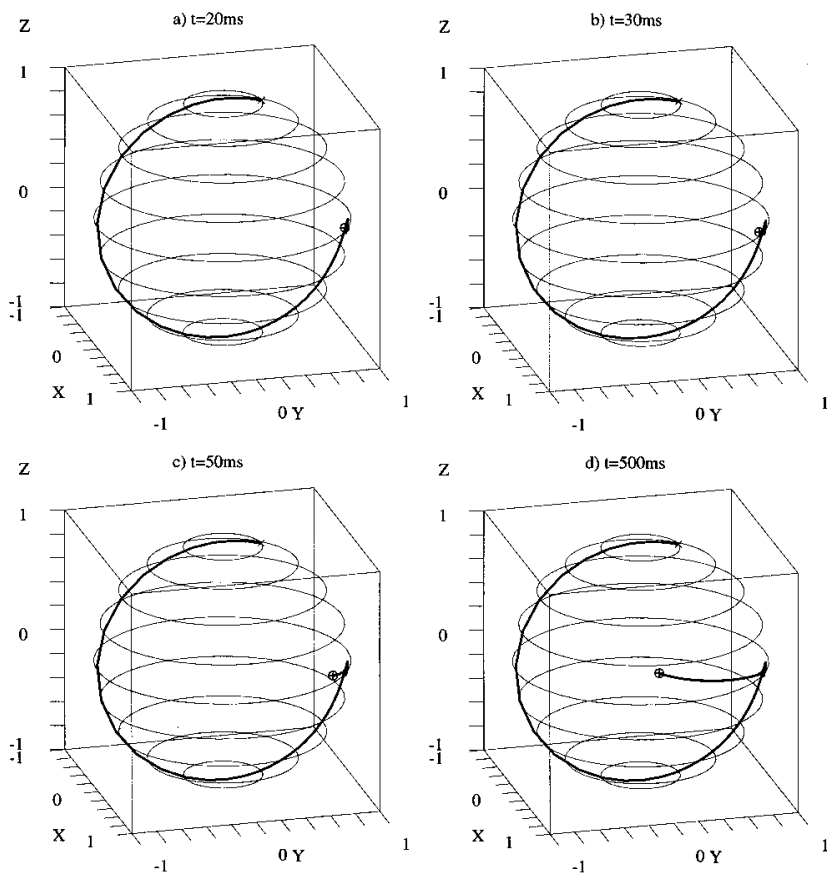

FIG. 3. The parameters used in the simulation are $\omega_{1}=300 \times 2 \pi \mathrm{rad} \mathrm{s}^{-1}$, $G=300 \times 2 \pi \mathrm{rad} \mathrm{s}^{-1}, T 1=3 \mathrm{~s}, T 2=400 \mathrm{~ms}, \delta=10 \mathrm{rad} \mathrm{s}^{-1}$. The evolution time varies from $30 \mathrm{~ms}$ to $500 \mathrm{~ms}$, from (a) to (d), respectively. The snapshots of the trajectory shown in (a) - (c) exhibit fairly good transient stabilization of the magnetization in the plane.

unrelaxed case, but is shifted by an amount which depends on the values of the parameters $\left\{\lambda, \delta, \omega_{1}\right\}$. It should be stressed again that this axis remains unchanged when varying the initial state of the magnetization. These results therefore suggest the possibility of performing self-calibrated selective radio-frequency pulses.

\section{EXPERIMENT}

Experimental realization of these selective pulses on the water resonance was achieved on a Bruker $600 \mathrm{MHz}$ spectrometer equipped with a commercial radiation damping control unit (RDCU) inspired by the authors' prototype. ${ }^{7}$ The pulse sequence used for the investigation of the magnetization dynamics is depicted in Fig. 4. It consists of a pseudo-2D experiment where an evolution period of

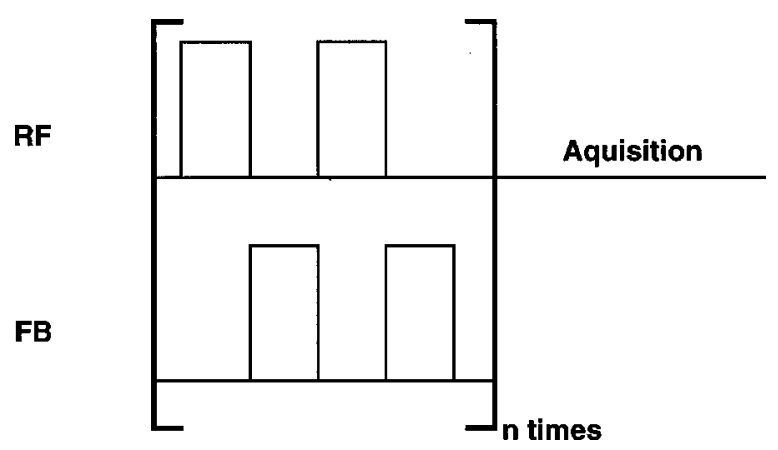

FIG. 4. Pulse sequence used in the experiments presented in this paper. A time sharing experimental scheme was used. Radio-frequency and feedback pulses are of $250 \mu$ duration. 


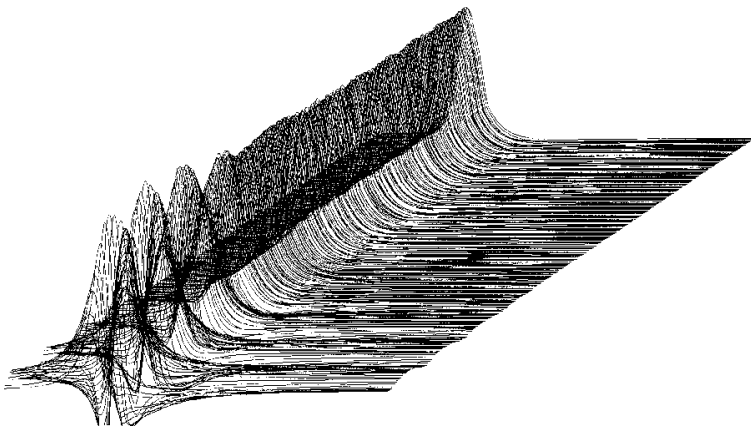

FIG. 5. Spectrum obtained in a typical pseudo-2D experiment corresponding to the pulse sequence of Fig. 4 performed on a sample of $90 \% \mathrm{H}_{2} \mathrm{O}-10 \% \mathrm{D}_{2} \mathrm{O}$. The initial evolution of the spectrum shows typical phase oscillation due to precession about a time-dependent axis before stabilization is reached. At longer times the spectra show good phase and amplitude stability, attesting for a transient but rather long period of stability (more than $75 \mathrm{~ms}$ ).

\{radio-frequency + feedback\} irradiation of increased duration was followed by a detection period. The acquired signal thus probed the evolution of the magnetization during the preceding evolution time, and the spectrum obtained for each increment of the evolution time is a point by point snapshot of the dynamics during irradiation. Since simultaneous radiofrequency and feedback irradiations are not allowed on these spectrometers, we had to resort to a kind of "time shared" irradiation scheme, where constant radio-frequency pulses alternate with feedback pulses. Pulse length was $250 \mu$ s for the experiments shown here. Experiments performed exhibit an initial oscillation of the phase of the water resonance, due to the precession of the magnetization about a timedependent axis, then followed by a quite long period of several tens of milliseconds (more than $50 \mathrm{~ms}$ in our experiments) where the resonance line has constant phase and constant amplitude, attesting for the stabilization of the precession axis. The intensity of the water resonance remains quasiconstant during this period. Indeed, a typical experiment is shown in Fig. 5, where the spectrum exhibits good stability for more than $75 \mathrm{~ms}$. A series of experiments where the initial magnetization was rotated by an arbitrary angle (from $0.3^{\circ}$ to $160^{\circ}$ ) gave exactly the same stabilization axis. The phase of the stabilization axis was determined to be steady during the experiment, and about $100^{\circ}$ with respect to the $\mathbf{B}_{1}$ axis, and completely reproducible from experiment to experiment. Calibration proceeded as follows. First, the phase of the feedback was adjusted so as to invert the water magnetization, as described elsewhere, ${ }^{9}$ and the intensity of the reinjected signal set to a value which yielded complete inversion in less than $10 \mathrm{~ms}$ in the absence of a $\mathbf{B}_{1}$ field. Note that the feedback intensity need not be calibrated, strictly speaking. Rather, its strength is chosen so that inversion of the magnetization is achieved in a time much shorter than both $T_{2}$ and $T_{1}$ in order to allow stabilization in the plane. This can be understood, in the light of the theory presented above, as setting experimental conditions which satisfactorily approximate the unrelaxed case. Then, pulse angle calibration was determined by systematically changing the radio-frequency intensity until the line intensity was equal to that obtained with a $\pi / 2$ hard pulse (for this calibra- tion, a long evolution period of about $100 \mathrm{~ms}$ was chosen to ensure stabilization of the axis). Let us add that we did not investigate the influence of the frequency offset on these experiments, and simply set the carrier of the spectrometer within the water resonance.

\section{DISCUSSION AND CONCLUSION}

In this paper, we have presented a theoretical analysis of the dynamics of the magnetization undergoing the simultaneous effect of a constant radio-frequency field and of a magnetization-slaved feedback field. It was found that under certain assumptions (the unrelaxed model) an asymptotic stable fixed point existed in the $x y$-plane, that is, the model predicted the possibility to perform slaved $90^{\circ}$ radiofrequency selective pulses. The above theory stimulated further study based on numerical simulation, which show that in the presence of relaxation, although there is no such fixed point in the $x y$-plane, one can find an analogous "stabilization" axis, along which the magnetization remains for periods of time of several tens of milliseconds, thus compatible with the realization of "slaved" selective pulses. Again, let us stress that slaved pulses are remarkable in that they do not rotate the magnetization by a certain flip angle, but they rather drive the magnetization to a desired direction in space. Moreover, these pulses can be thought of as self-calibrated with respect to flip angle and phase. Indeed, pulse length calibration of conventional selective pulses is crucial, and it is well known to every NMR spectroscopist that any deviation of the magnetization vector from its expected initial position in space or any deviation of the pulse duration from the one exactly necessary to yield complete inversion or exact $90^{\circ}$ rotation onto the $x y$-plane, respectively, results in a loss of accuracy of the pulse. In contrast, when slaved pulses are used, the magnetization ends up aligned parallel to the equilibrium direction in space (although for a limited but still long period of time), which can be made the $z$ axis, or any axis in the $x y$-plane, depending on the parameters $\left\{\lambda, \delta, \omega_{1}\right\}$. And for the same reason, there is also no phase calibration of the pulse. Experimental demonstration was achieved by performing actual experiments on a water sample. Indeed, the magnetization was found to reach the $x y$-plane, where it remained stable (conservation of intensity and phase) for more than $75 \mathrm{~ms}$. However, the theory outlined in this paper remains to be developed further, and extension along with applications of the experiments presented here to more involved pulse sequences, and to particularly suitable applications such as water-solute interactions need be investigated. Both these aspects are currently under study.

\footnotetext{
${ }^{1}$ M. A. Mc Coy and W. S. Warren, J. Chem. Phys. 93, 858 (1990).

${ }^{2}$ N. Bloembergen and R. V. Pound, Phys. Rev. 95, 8 (1954).

${ }^{3}$ G. Deville, M. Bernier, and J. M. Delrieux, Phys. Rev. B 19, 5666 (1979).

${ }^{4}$ D. Abergel and J. Y. Lallemand, J. Magn. Reson., Ser. A 110, 45 (1994).

${ }^{5}$ A. Vlassenbroek, J. Jeener, and P. Broekaert, J. Chem. Phys. 103, 5886 (1995).

${ }^{6}$ J. Jeener, A. Vlassenbroek, and P. Broekaert, J. Chem. Phys. 103, 1309 (1995).

${ }^{7}$ A. Louis-Joseph, D. Abergel, and J. Y. Lallemand, J. Biomol. NMR 5, 212 (1995).
} 
${ }^{8}$ P. Broekaert and J. Jeener, Magn. Reson. Ser. A 113, 60 (1995).

${ }^{9}$ D. Abergel, A. Louis-Joseph, and J. Y. Lallemand, Chem. Phys. Lett. 262, 465 (1996)

${ }^{10}$ For an introduction to the mathematics of nonlinear systems, many general references are available. See, for instance, D. K. Arrowsmith and C. M. Place, Dynamical Systems: Differential Equations, Maps, and Chaotic
Behavior (Chapman \& Hall, New York, 1992).

${ }^{11}$ In fact, this is true only for simple fixed points, for which $\operatorname{det}(\partial V) \neq 0$, which means that in the two-dimensional case the linearized system has two strictly nonzero eigenvalues. The case of nonsimple fixed points can be much more complicated, and a discussion is beyond the scope of this paper. 